\title{
Cuando lo técnico se torna político: un análisis del proyecto de modificación excepcional del Plan de Ordenamiento Territorial de Bogotá a partir del marco de coaliciones promotoras
}

Adriana-Marcela Sánchez-Ospina. Pontificia Universidad Católica de Chile, Santiago, Chile.

Sebastián Hernández-Mora. Universidad Nacional Autónoma de México, Ciudad de México, México.

RESUmen | El Plan de Ordenamiento Territorial es una política pública de los municipios colombianos, que a través de un conjunto de directrices delimita instrumentos de planeación, gestión y financiación. Sin embargo, para que se implemente es necesario que el Concejo Distrital, un órgano político, lo apruebe. Este artículo analiza, a partir de entrevistas, actas del Concejo y revisión de la prensa, las creencias y posiciones de los actores políticos durante el trámite de modificación excepcional del Plan de Ordenamiento Territorial presentado al Concejo de Bogotá el año 2013, utilizando metodológicamente el marco de coaliciones promotoras. Se concluye que, pese a ser un proyecto técnico, la discusión alrededor del mismo se tornó política en el Concejo, por las relaciones tensas entre el gobierno nacional y el municipal y por la presión de los grupos de interés, que produjeron como resultado el archivo del proyecto normativo.

PALABRAS CLAVE | gobierno local, política urbana, ordenamiento territorial.

ABstract | The Master Plan of Bogotá is a public policy of the Colombian municipalities, because of its guidelines for planning, management, and financing. However, in order for the plan to be implemented, it is necessary for the municipal council (a political body) to approve the instrument. This article - which is based on interviews, and law and press review - analyses the beliefs and positions of the political actors during the process of exceptional modification of the master plan submitted to the Bogota municipal council in 2013, using the model of advocacy coalitions framework. It is concluded that, despite being a technical project, the discussion with the council became political, due to tense relations between the national and municipal government, and due to the pressure of the interest groups that resulted in the archiving normative project.

KEYWORDS | local government, urban policy, spatial planning. 


\section{Introducción}

Desde la promulgación de la Ley 388 de 1997, es obligatorio en Colombia, sin importar la cantidad de población o el tamaño de los municipios, que estos elaboren Planes de Ordenamiento Territorial, definidos como el conjunto de objetivos, directrices, políticas, estrategias, metas, programas, actuaciones y normas adoptadas para orientar y administrar el desarrollo físico del territorio y la utilización del suelo (Congreso de Colombia, Ley 388 de 1997, artículo 9², Plan de Ordenamiento Territorial). Estos planes son el principal instrumento de planeación de los municipios colombianos y, según la legislación nacional, deben ser elaborados por la Alcaldía municipal, concertados con la autoridad ambiental, discutidos con la comunidad y aprobados por el Concejo municipal. Este último es un cuerpo político, escogido democráticamente cada cuatro ańos, vía representación de partidos, movimientos políticos o movimiento significativo de ciudadanos.

Los Planes de Ordenamiento Territorial (РОт) definen, entre otros aspectos, la clasificación y las actividades económicas del suelo rural y urbano de los municipios; es decir, delimitan las zonas de crecimiento de la ciudad y las zonas de protección ambiental donde se puede prohibir parcial o totalmente el desarrollo inmobiliario; fijan instrumentos de gestión y financiación del suelo, como los planes parciales (desarrollo o expansión urbana); y reglamentan las cargas y obligaciones urbanísticas. Por ello estos instrumentos adquieren un carácter relevante para la ciudad, al fijar una ruta para el crecimiento físico y económico en el territorio municipal.

Bogotá adoptó su primer Plan de Ordenamiento el año 2000, lo modificó en 2003 y lo compiló en el Decreto 364 de 2004, norma vigente. Intentos de posteriores alcaldías buscarían introducir modificaciones al instrumento, pero solo en 2013 se concretó la iniciativa. Ello es posible dado que, a pesar de la vigencia legal de los рот, se pueden introducir modificaciones a su contenido. La ley reglamenta que estos tienen una temporalidad de doce años (actualmente, tres períodos de gobiernos locales); sin embargo, se permiten hacer modificaciones al рот -de carácter general o específico- cada cierto tiempo.

Específicamente, el Decreto Nacional 4002 de 2004, en su artículo 6º permite que los municipios introduzcan modificaciones excepcionales a las normas urbanísticas de los planes de ordenamiento en cualquier momento a "iniciativa del alcalde municipal o distrital, siempre y cuando se demuestren y soporten técnicamente los motivos que dan lugar a su modificación" (Presidencia de la República, 2004). Ello quiere decir que no se pueden modificar los objetivos de largo plazo del рот, ni se puede hacer una revisión general a su contenido o hacer uno nuevo, sino que las modificaciones excepcionales deberán hacerse con la finalidad de cumplir con los lineamientos del РОт vigente.

Con base en lo anterior, cinco motivaciones consideró en su momento la alcaldía de Gustavo Petro (2012-2015) para presentar una modificación excepcional ante el Concejo: i) hay cambios en las proyecciones y composición poblacional de la ciudad; ii) es necesario introducir nuevos proyectos de alto impacto en la movilidad de la ciudad; iii) debe integrarse al ordenamiento territorial tanto la gestión del riesgo como la adaptación al cambio climático; iv) es necesario armonizar el 
ordenamiento del suelo rural con la legislación nacional; y, finalmente, v) hay que simplificar normas relacionadas con los usos de suelo, hacer claridad en la aplicación de los tratamientos urbanísticos y reconfigurar la edificabilidad en función de la capacidad de soporte urbano de cada zona de la ciudad.

Después de un proceso de concertación con la Corporación Autónoma Regional (CAR), de socializar el proyecto y las modificaciones con las localidades y recibir el concepto del Consejo Territorial de Planeación Distrital (CTPD), el gobierno local trasladó el proyecto al Concejo en mayo de 2013. En doce sesiones de la Comisión del Plan de Desarrollo y Ordenamiento Territorial fue abordado el proyecto, y en junio de ese ańo sería rechazado por el Concejo, tan solo un mes después de que fuera radicado. ¿Por qué se rechazó? ¿Cuáles fueron las motivaciones técnicas y/o políticas para archivar el proyecto? Este artículo argumenta que en las discusiones dentro y fuera del Concejo se visibilizaron intereses no solo políticos, sino también económicos, en la toma de decisiones públicas, intereses que evidencian la postura de los actores sobre cómo debe ser producida la ciudad. Por lo anterior, independientemente de que se pudiera estudiar la conveniencia para la ciudad de la propuesta de Modificación excepcional del Plan de Ordenamiento Territorial de Bogotá (мерот), este artículo identifica y analiza las posiciones y las creencias de los actores políticos que motivaron el rechazo de la modificación a la normativa. Para cumplir con lo anterior, el texto se divide en cuatro partes, además de esta introducción: i) se explica el modelo de coaliciones promotoras, aspecto metodológico de esta investigación; ii) se describe la formación de coaliciones y creencias de los actores; iii) se analiza la discusión política alrededor del proyecto; y, finalmente, iv) se presentan algunas consideraciones finales.

\section{Coaliciones promotoras y la metodología de trabajo}

Coaliciones promotoras o advocacy coalition framework (ACF) es un enfoque diseñado por Sabatier y Jenkins-Smith en la década de los ochenta y posteriormente modificado y perfeccionado por Sabatier y Weible (2007, 2010). No es propiamente una teoría, y sí un método de análisis para entender el proceso político y visibilizar el sistema de creencias de los actores que elaboran políticas públicas e identificar cambios institucionales en periodos de largo plazo. Debido a su versatilidad, el marco ha sido ampliamente usado en la literatura como una forma de acercamiento a las dinámicas de construcción y cambio en políticas públicas en ámbitos diferenciados. Los más recientes estudios latinoamericanos apuntan a una expansión de sus horizontes hacia temas que sus autores originales no exploraron, destacando la aplicación del enfoque en análisis de programas de desarrollo rural (Meglio \& Pluchino, 2019); políticas de drogas (Labiano, 2018; Sanjurjo García, 2013); políticas de educación media (Pérez Zorrilla, 2016); políticas socioambientales (Cortez \& Maillet, 2018); y políticas de atención a víctimas (Hernández Mora, 2019), entre otros.

Dada la complejidad inherente a la formulación de políticas públicas, entendidas estas como el Estado en acción, y especialmente en cuanto a determinar cómo se hacen y quiénes toman las decisiones, los autores del enfoque asumen que hay un 
nivel de especialización dentro del campo denominado subsistema, compuesto por un conjunto de actores que buscan influir en la política que lo rige; por tanto, el subsistema se caracteriza por tener una dimensión funcional/sustantiva (en nuestro caso, la política urbana) y una territorial (Bogotá, siguiendo el caso de estudio) (Sánchez Ospina, 2019).

El marco de las coaliciones promotoras es definido bajo el supuesto de que las personas involucradas en política buscan trasladar sus creencias, más allá de simples intereses materiales, a acciones concretas; y, por tanto, las coaliciones se forman como grupos de actores con diferentes sistemas de creencias que interactúan y compiten para dominar un determinado subsistema de políticas (Cairney, 2016, p. 485). Adicionalmente, el marco se centra en la interacción entre las coaliciones en disputa en un subsistema de políticas que, a su vez, opera dentro de un sistema político más amplio influido por eventos externos (2016, p. 485), pero también internos de las mismas coaliciones, por aprendizaje de políticas (policy learning) y acuerdos negociados entre sus integrantes (Pierce, Peterson \& Hicks, 2017, p. 2). El modelo, de igual forma, asume que hay creencias normativas preexistentes sobre la política (politics) que son difíciles de modificar y, por tanto, la información recibida por los actores puede ser interpretada de modos diferentes; considerando lo anterior, las coaliciones son manifestaciones de comportamiento de ideas, valores y compromisos ideológicos comunes (Sánchez Ospina, 2019).

Ahora bien, las creencias de los actores se agrupan en tres subniveles:

- Un nivel fundamental de creencias de núcleo duro (deep core beliefs), definidas como valores axiológicos, ontológicos e ideológicos de los actores; tales creencias son difíciles de modificar en el corto plazo y se manifiestan en todos los subsistemas de políticas, funcionando como una filosofía personal profunda (Jenkins-Smith, Silva, Gupta \& Ripberger, 2014, p. 485).

- Un segundo nivel, donde se encuentran las creencias propias asociadas a la política pública (policy core beliefs), en las que se incluyen las percepciones de la naturaleza del problema público que se requiere atender y la posibilidad de que sea resuelto; también incluye las consideraciones respecto del papel del gobierno y del mercado en dicho proceso, así como la distribución de poder en los diversos niveles de gobierno (2014, p. 485). Creencias de este tipo son modificables gracias a aspectos exógenos al subsistema de políticas.

- El tercer nivel de creencias de carácter instrumental (secondary beliefs) está relacionado con aspectos secundarios de la política pública; no necesariamente aborda todos los aspectos del subsistema, pero sí algunos de ellos. Estas creencias se manifiestan en la discusión de cualquier política pública como elementos técnicos y negociables. Los actores que comparten estos aspectos forman coaliciones, buscando que sus creencias sean incorporadas en las políticas públicas. 
FIGURA I | Estructura del sistema de creencias en un subsistema de política

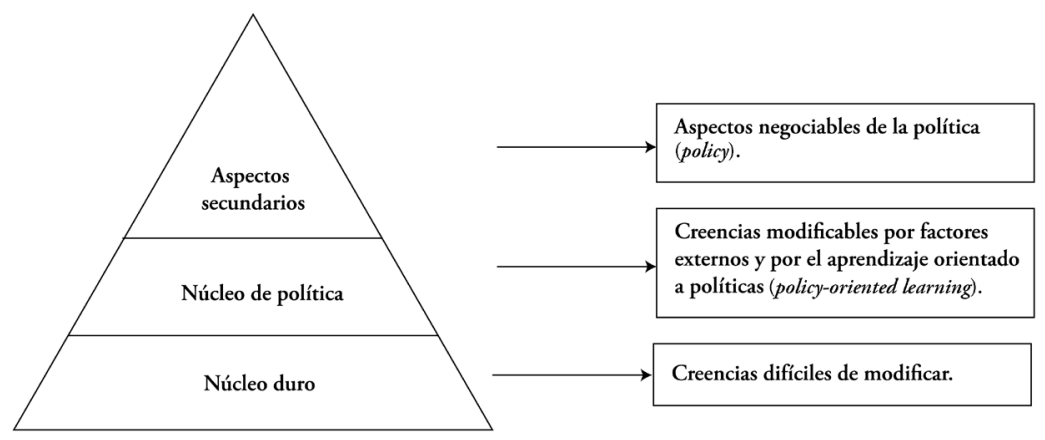

FUENTE: ELABORACIÓN PROPIA

En un subsistema de política cualquiera, dos o más coaliciones visibilizan sus creencias y las posicionan para que sean aprobadas. Sin embargo, señalan Sabatier y Jenkins (1999), eventos externos e internos en el propio subsistema pueden afectar (o no) el cambio de una política pública. Entre los eventos externos están la formación de coaliciones políticas y cambios en las condiciones socioeconómicas y en la opinión pública. Entre los internos, la (in)experiencia de los actores sobre la discusión específica de la política, la capacidad de persuadir a los actores con la información disponible, y el factor cognitivo, que tiene un papel relevante en la modificación de las creencias de los participantes. Por tanto, los especialistas en el área (académicos y consultores) asumen un rol principal en el proceso de la política, al inducir la discusión para su modificación o continuismo. Este aprendizaje (policyoriented learning) tiende a provocar una alteración en las creencias de los actores, la cual es resultado de la dinámica de los debates que tienen lugar en torno a los conflictos entre las coaliciones (Figura 1).

Con base en lo anterior, esta investigación es un estudio de caso sobre la modificación al Plan de Ordenamiento Territorial de Bogotá a partir del modelo de coaliciones promotoras. Se trata de un estudio empírico sobre la formación de coaliciones (y el conjunto de creencias que comparten sus integrantes), constituidas para que sea modificada (o no) la política pública. A fin de identificar las creencias, recursos y estrategias de los actores para la aprobación (o no) de esta política, y también para el análisis de la información, se utilizaron varias herramientas metodológicas. Se partió de una recopilación de las actas y sesiones en el Concejo de la ciudad sobre la discusión del proyecto de modificación excepcional del рот; a ello se sumó la realización de cinco entrevistas a actores políticos, académicos y de grupos de interés que participaron en esas sesiones, y el registro en prensa de dichas reuniones. 


\section{Creencias y posiciones de los actores para formar coaliciones promotoras}

En 2013, año en que fue discutido el proyecto de Modificación excepcional del Plan de Ordenamiento Territorial de Bogotá en el Concejo de la ciudad, la Administración Distrital ya tenía una difícil relación con la Corporación; sin embargo, la mayoría de los proyectos relevantes hasta entonces habían sido aprobados en las comisiones y en el pleno. ${ }^{1}$ No obstante, este proyecto, мерот, que se iría a discutir entre mayo y junio de ese año, agudizó la tensa relación entre el Concejo y la Administración Distrital, y entre esta última y el sector privado. Ello se debe a las posiciones y creencias contrarias entre los actores por la implementación de la norma.

Desde una de las perspectivas en juego, se tiene que la Ley 388, los decretos nacionales sobre promoción de vivienda social, el рот del año 2000 у su compilación en 2004 reafirmaban -según señala Sánchez (2019)- un conjunto de creencias sobre la política de ordenamiento territorial, que se resumen en que si el Estado interviene, debe generar incentivos para la construcción de vivienda con la finalidad de que el privado asuma su promoción. En este marco, se sostiene: i) que dicha política debe impulsar una distribución equitativa de cargas y beneficios urbanísticos; ii) la obligatoriedad de construcción de Vivienda de Interés Prioritario (vIP), la cual debe operar vía incentivos para el sector privado; iii) que la delimitación de zonas que se destinen a la renovación urbana no debe impedir o limitar la construcción de vivienda en zonas de expansión urbana; y iv) que las condiciones para crear y/o aumentar la edificabilidad deben ser incentivos para el promotor, no promover obstáculos.

Estas creencias, que son propias de la intervención del Estado en la producción del capital, son presunciones de largo plazo y se deben a que -como reseñan Jaramillo y Cuervo (2014) - a partir de los años ochenta en Colombia, el promotor de la vivienda pasa a ser el sector privado, mientras el Estado modifica su rol y lo sustituye por entregar subsidios directos al consumidor. Desde esta lógica, la vivienda social es incentivada a través de los subsidios a la demanda; y siguiendo al mercado y las economías de escala, se localiza en la periferia. Consecuencias de este tipo de producción han sido analizadas en América Latina a través de los estudios de segregación socioespacial y distribución inequitativa de los servicios de la ciudad (Marques \& Kowarick, 2013; Sabatini \& Brain, 2008). No obstante, los planes de ordenamiento territorial siguen promoviendo este tipo de producción de vivienda. En ese sentido, como plantea Leal (2002), el mercado de vivienda es uno de los mecanismos de distribución de los hogares según sus características sociales, sus ingresos y su patrimonio, siendo imposible comprender la segregación sin entender la manera en que actúa el mercado de vivienda en cada ciudad.

Como se observa, esta producción de ciudad tiene una explicación ideológica (deep core beliefs), que es reseñada por Jaramillo y Cuervo (2013, pp. 13-14) al señalar que, dada la ineficiencia y la burocracia de los organismos estatales, en las

Entre los proyectos aprobados por el Concejo se encontraban la creación de la Secretaría de la Mujer, la aprobación del Plan de Desarrollo, y la expedición del presupuesto de 2013. No obstante, la Corporación no le aprobó al alcalde la propuesta de modernización tributaria que presentó dos veces en 2012 y que buscaba modificar la tarifa predial de la vivienda en la ciudad. 
que buena parte de los recursos públicos se desvían (por corrupción) o se desperdician (no se destinan a los usuarios más necesitados), se recurre a los subsidios directos al usuario, de tal manera que los habitantes más pobres puedan acceder a la demanda. En ese sentido, lo que antes producía una entidad estatal ahora será atendido por empresas, en libre competencia, lo cual -se supone- hará más eficiente la administración pública, que cooperará con el emprendimiento inmobiliario y con el sector privado.

La otra corriente en juego en el marco del proyecto de Modificación excepcional del Plan de Ordenamiento Territorial de Bogotá tiene que ver con la financiación de los servicios de la ciudad. Si bien la Constitución política y la Ley 388 de 1997 hicieron avances significativos sobre la regulación de la propiedad y han contribuido a la obligatoriedad de la promoción de vivienda social por parte del sector privado, frente al tema de la financiación para construir los soportes de la urbanización (parques, vías, equipamiento, redes de servicios públicos), la situación ha sido más difícil. Como señala Sánchez (2019), en la lógica del mercado y la producción de la ciudad neoliberal, es necesario exceptuar impuestos para atraer el desarrollo, u otorgar mejores aprovechamientos a los suelos a fin de generar inversiones. Además, el gremio constructor asume una posición en la cual las cargas asignadas a la urbanización son impuestos, y no ingresos no tributarios no constitutivos de precio; es decir, que no son asumidos como contraprestación que adquiere el suelo gracias a decisiones colectivas.

$\mathrm{Al}$ introducirse la мерот, tres cuestiones colocaron en consideración los actores partícipes que buscaban modificaciones al ordenamiento vigente: i) debe haber obligatoriedades para la construcción de Vivienda de Interés Prioritario (VIP) en el centro ampliado; ${ }^{2}$ ii) es necesaria la adquisición de suelos destinados a incrementar la provisión de parques, equipamiento, espacio peatonal y sistemas viales; y iii) el desarrollo de los sistemas de acueducto y alcantarillado. Como se observa, son lineamientos opuestos a la forma como se produce la ciudad actualmente.

Para la administración distrital, lo anterior era justificable a partir del cambio demográfico de la ciudad, pues de acuerdo a cálculos del Departamento Administrativo Nacional de Estadística (DANE), el número de habitantes en la ciudad ha disminuido en los últimos quince años, afirmación que se sustenta, en primer lugar, en que las cifras de crecimiento de población utilizadas para elaborar el РОт de 2000 habrían sido sobreestimadas. Un análisis de estas cifras indica que, para realizar ese РОт, se utilizaron las proyecciones de demanda de agua realizadas en 1997 por la Empresa de Acueducto y Alcantarillado de Bogotá (EAAB), basadas en los resultados finales del Censo de Población de 1993, publicados ese mismo año. En dichas proyecciones se estimaba para el año 1999 una población de 6.322 .702 habitantes, y 8.086.532 para el año 2010, lo que suponía un incremento de población entre dichos años de 1.763 .830 personas; es decir, aproximadamente 160.348 nuevos habitantes por ańo, a una tasa de crecimiento promedio anual de 2,2\% (Secretaría

2 El centro ampliado es un concepto administrativo creado durante la alcaldía de Gustavo Petro, que comprendía entre la avenida Primero de Mayo y calle Ochenta y entre la carrera Séptima y la avenida Boyacá, ocupando un total de 11.000 hectáreas, donde se concentran las mayores actividades administrativas y financieras de la ciudad. 
de Planeación, 2013, p. 9). Posteriormente, con la realización del Censo Nacional de 2005, el DANE estimó una población oficial para ese año de 6.840.116 (DANE, 2013), lo que mostró que había disminuido a $1,48 \%$ el crecimiento poblacional anual (Secretaría de Planeación, 2013).

En razón de lo anterior, y de las proyecciones municipales de 1985 a 2020 que realizó el DANE con base en el Censo de 2005, la tasa de crecimiento promedio anual, que para el periodo 2000-2005 había sido estimada por la ЕAAB en 2,32\%, descendió, según la proyección del DANE, a 1,68\% anual. Por lo tanto, para el caso de los cálculos del РОт del año 2000 se habría sobreestimado la población en 577.979 nuevos pobladores, con evidentes impactos en el ordenamiento territorial de la ciudad (Secretaría de Planeación, 2013). Por otro lado, siguiendo las proyecciones del DANE, la tasa de crecimiento de la ciudad para el 2011 (ańo en que se comenzaron a hacer los estudios técnicos de la modificación excepcional) fue de $1,40 \%$, estimándose una población total de 7.467.804; es decir, que la población estaba aumentando, pero no a un ritmo tan acelerado como se había considerado en el anterior рот. Ello también se refleja en las proyecciones del DANE alrededor de la tasa de fecundidad, pues el número de hijos que en promedio han tenido las mujeres residentes en Bogotá ha ido disminuyendo progresivamente, pasando de una tasa global de fecundidad estimada de 1,91 entre 2010 y 2015, a una de 1,9 en el quinquenio 2015-2020; es decir, que en promedio una familia estaba teniendo menos de dos hijos.

En segundo lugar, a partir de los años noventa la tasa de migración también había disminuido en Bogotá, y ello debido a menores costos de habitación y la facilidad de conseguir trabajo en labores de mano de obra no calificada en los municipios vecinos, en oposición a los altos precios de los alquileres y la compra de vivienda en la ciudad, lo que se refleja en las tasas de crecimiento poblacional de los municipios circunvecinos (Secretaría de Planeación, 2013). Finalmente, la composición de los hogares también se había modificado, pues en la ciudad se estaban formando cada vez más hogares monoparentales, mostrando que, según la encuesta multipropósito de la ciudad del año 2011 (Secretaría de Planeación, 2011), de los 2.185 .872 hogares urbanos monoparentales, en un 34,8\% (759.595) eran de jefatura femenina, con el restante $65,2 \%$ (1.426.279) de jefatura masculina. De igual forma, la encuesta mostraba que, de ese total de hogares urbanos, el $15,1 \%$ se encontraba en situación de pobreza.

A partir de lo anterior, la administración distrital proponía ajustar las áreas de localización de las viviendas con el objetivo de controlar los procesos de expansión urbana, lo que, a su vez, podría ser útil para mejorar la calidad de vida de las personas en condición de pobreza. Con ello se introducen dos lineamientos para vivienda social: i) dado que había sido reglamentada una obligación urbanística por el Decreto Nacional 075 de 2013, la cual obligaba a definir los porcentajes mínimos de suelo útil destinado a la producción de Vivienda de Interés Prioritario, 
la modificación excepcional proponía que en los predios objeto de tratamiento urbanístico $^{3}$ de desarrollo se destinara un mínimo de 30\% del área útil con uso residencial a la producción de Vip, y que en los predios objeto del tratamiento urbanístico de renovación urbana por redesarrollo, la obligación sería del $20 \%$ sobre el total del área útil del proyecto; y ii) la modificación introduce la edificabilidad condicionada, esto es, que a partir del índice de construcción base para acceder al índice de construcción máximo que plantea la norma, la edificabilidad adicional está condicionada a la producción de vip. En esa línea, solo es posible acceder a edificabilidades mayores a dos siempre y cuando se genere viP.

Para la oposición, las cifras entregadas por la administración distrital y que tenían validez a partir de los estudios censales del DANE eran insuficientes, pues todavía eran necesarios estudios "científicos" para validar los ajustes poblaciones:

Mire, yo no sé cómo miden esos cambios poblacionales. No podemos tener la certeza de un cambio poblacional en Bogotá (...). Entonces, sustentar un motivo en proyección -en mi criterio- es antitécnico; y en segundo lugar, antitécnico porque no tiene bases científicas, tiene base estadística. Pero, segundo, es ilegal, porque es un elemento sustancial para cambiar el modelo de largo y mediano plazo (...). Se convierte en una confesión simple de un cambio de modelo de ciudad. (Juan Manuel González, abogado urbanista, asesor de Camacol, sesión del Concejo del 24 de mayo, 2013)

Con las introducciones hechas en la мерот se podrían crear nuevas obligatoriedades para la vivienda social y prioritaria dentro de los planes parciales, especialmente promover la vivienda social en el centro ampliado:

Desde esa perspectiva de lo que estamos planteando, es: invirtamos con un sistema de estímulos y desestímulos a la ciudad, a que traiga y a que se genere la vivienda en las áreas que tienen menor densidad de las zonas centrales, y desestimulan la localización sobre esas zonas que están siendo sujetas a procesos de riesgo en muy poco tiempo. ¿Cómo lo hacemos? De la única manera en que podemos hacerlo: limitando las edificabilidades en las zonas de borde; limitando las posibilidades de grandes construcciones y, además, concentración de viviendas en esas zonas; y generando estímulos en las zonas centrales. [Intervención de Gerardo Ardila, secretario de Planeación] (Concejo de Bogotá, 2013)

Por el contrario, esta modificación fue asumida por el sector constructor como una carga urbanística, que desincentivaría la construcción y aumentaría el precio del suelo, alza que sería trasladada al usuario final. De allí su influencia y participación

3 Los tratamientos urbanísticos orientan las intervenciones en el territorio mediante una respuesta diferenciada, según características físicas de cada zona, para consolidar, mejorar, revitalizar los espacios físicos del municipio. En Bogotá se aplican cinco: conservación para proteger el patrimonio construido; mejoramiento integral, para las regularizaciones de origen informal; consolidación, para afianzar transformaciones en la ciudad desarrollada; desarrollo, aplicado a las zonas de expansión urbana; y renovación urbana, para mejorar zonas que tienen condiciones de subutilización. 
en las sesiones del Concejo. Así lo reseña el informe del Centro de Estudios Económicos de la Asociación Nacional de Instituciones Financieras (ANIF, 2013):

Por ejemplo, las exageradas cargas urbanísticas a los constructores amenazan con encarecer el precio de venta final de los proyectos, en una ciudad como Bogotá, que ya ostenta el penoso tercer lugar en encarecimiento y caos urbanístico, después de São Paulo y Rio de Janeiro. También cabe recordar que propuestas similares de redensificación fueron implementadas en Shenzhen, cerca de Hong Kong (China), las cuales resultaron en fracasos. Allí surgieron los denominados "handshake buildings": edificios de 10 pisos con menos de un metro de espacio entre ellos; única forma de hacerlos viables económicamente. (p. 1)

Este lineamiento frente a la modificación de la localización de la vivienda va de la mano con el ajuste de los programas frente al ordenamiento ambiental de la ciudad. Específicamente, frente a la estructura ecológica principal, la administración planteaba introducir nuevas áreas de protección ambiental y limitar la construcción en zonas de alto riesgo, introduciendo límites a la urbanización del Plan Zonal Norte o a proyectos urbanos específicos, como Campo Verde en la localidad de Bosa. Las estrategias ambientales iban dirigidas en dos sentidos: medidas de mitigación, que se harían implementando políticas de movilidad, como el incentivo al transporte público, la ampliación de las ciclovías e inclusive eliminando la obligatoriedad de los cupos de parqueaderos para los nuevos proyectos de vivienda; y, por otro lado, medidas de adaptación, con la localización de vivienda social en centralidades y la prohibición de actividades extractivas en el municipio.

Para la oposición, esto significaba limitar la actividad económica de la ciudad, crear especulación inmobiliaria, limitar los desarrollos inmobiliarios en curso e, inclusive, modificaba el modelo de la ciudad, pues el alcalde estaba facultado para introducir modificaciones al рот vigente, no para modificar los objetivos de largo plazo del ordenamiento territorial.

Ninguna de las dos coaliciones presentó un estudio técnico ante el Concejo sobre aumento o disminución del precio del suelo si aumentaban las cargas urbanísticas, pero sí recurrieron a teorías económicas para justificar la aprobación o no de las modificaciones; es decir, para los actores, la política de vivienda se constituye de forma institucional a partir de posturas ideológicas y políticas. Trasladar esas posiciones ideológicas y políticas a la aprobación o rechazo de la iniciativa, fue el núcleo central en la discusión de la мерот. A partir de lo anterior, es posible identificar dos coaliciones promotoras en la discusión de este proyecto: ${ }^{4}$ por un lado, una coalición conformada por el gobierno municipal, el gremio de la microempresa (Asociación Colombiana de las Micro, Pequeñas y Medianas Empresas, ACOpI), y el sector

Se puede identificar una subdivisión en la coalición pro modificación dado que, si bien los actores querían que se aprobase la iniciativa normativa, algunos pedían modificar algunos artículos. Para fines de investigación, como nos enfocamos en las creencias del núcleo duro y del núcleo de políticas, se opta por agregarlos en la misma coalición. 
académico que participó en el Concejo ${ }^{5}$ y en su opuesto, una coalición conformada por los gremios asociados al sector constructor, de materiales de construcción y de comercio, como la Cámara Colombiana de la Construcción (Camacol), la lonja de propiedad raíz de Bogotá, la Asociación Nacional de Fabricantes de Ladrillo (Anafalco) y la Federación Nacional de Comerciantes (Fenalco); por los concejales que eran de oposición al gobierno local; y por el gobierno nacional a través del ministro de Vivienda y los órganos de control.

Para fines de esta investigación, la primera coalición se ha denominado "coalición promotora pro modificación", dada sus aspiraciones para instaurar nuevos lineamientos para el ordenamiento físico del municipio; y la segunda, "coalición promotora statu quo", pues si bien algunos actores compartirán creencias instrumentales de la primera coalición, su objetivo principal es impedir la aprobación de las modificaciones excepcionales. Como se observa, este proyecto discutiría en su conjunto el cómo de la producción de la ciudad (Tabla 1).

En la coalición promotora pro modificaciones, los actores que la conformaban eran locales, mientras la coalición promotora statu quo estaba conformada por actores multisectoriales y nacionales, lo que imprimiría un carácter nacional a la discusión del proyecto en la corporación local. La consecuencia de esto es el debate nacional que fue registrado en la prensa, televisión y radio sobre el ordenamiento territorial de la ciudad.

Junto con las posiciones señaladas, también es posible identificar las creencias de los actores intervinientes respecto de un proyecto político. En esta perspectiva, es importante señalar que los actores que hacían parte de cada una de las coaliciones compartían un proyecto ideológico. Por ejemplo, los concejales que se oponían a la modificación del рот significativamente pertenecían a partidos políticos de derecha, pero incluso quienes no se reconocían bajo esta posición política, eran opositores a alcaldías de izquierda:

Yo ya tenía una predisposición a lo que venían haciendo los gobiernos de izquierda en Bogotá (...) yo sí creo que Lucho, Samuel y Petro le dieron forma a un clientelismo de izquierda muy fuerte. (Fernando Rojas, asesor del concejal Juan Carlos Flórez, com. pers., 24 de julio de 2018)

Por su parte, los concejales que compartían las iniciativas tendientes a la modificación del рот venían de militar o formar parte de partidos o movimientos de izquierda en Bogotá, o incluso nacionales. Por consiguiente, es posible identificar posiciones políticas en las creencias de los actores que, de hecho, influirían en el tránsito del proyecto normativo.

Dado el objetivo del artículo, se omite analizar el proceso de participación que tuvo lugar antes de la discusión del proyecto de acuerdo en el Concejo; sin embargo, como lo manifiesta el Consejo Territorial de Planeación Distrital, varios miembros de la comunidad no estaban de acuerdo con las modificaciones hechas por la administración. De ello queda constancia en las actas de este Consejo en las memorias de la мерот (2013). 







\section{Lo técnico y la política}

La Modificación excepcional al Plan de Ordenamiento Territorial es esencialmente un proyecto técnico. La administración, por ley, debe presentar una memoria justificativa, el proyecto normativo, la planimetría, y principalmente los soportes técnicos que justifiquen introducir una modificación. Una vez socializados los documentos y seguir el proceso de formulación, el 19 de mayo de 2013 el proyecto comenzó su curso normativo, y el 7 de junio se celebró la votación: nueve votos contra seis negaron el proyecto de acuerdo.

Al observar la discusión del proyecto en el Concejo, es posible señalar cómo las creencias de ambas coaliciones serían abordadas en la corporación y cómo, ante todo, el proyecto se transformó en un asunto político. El modelo de coaliciones promotoras permite hacer el ejercicio de extrapolación (Figura 2):

FIGURA 2 | Modelo de coaliciones promotoras a la мерот

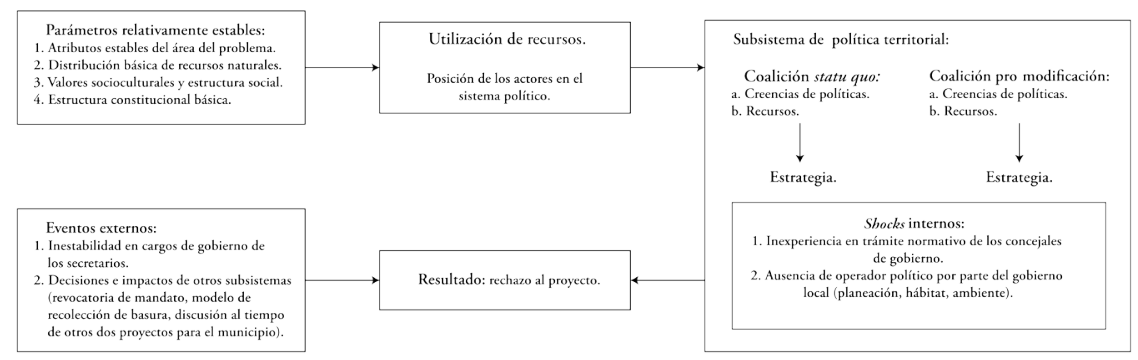

FUENTE: ELABORACIÓN PROPIA

Por un lado, es posible identificar los recursos con los que contaban ambas coaliciones y cómo, a partir de ellos, se establecieron las estrategias para posicionar las creencias. Específicamente, ambas coaliciones recurrieron a los medios de comunicación con la finalidad de trasladar el debate hacia la ciudadanía, buscando declarar (i)legítimo el plan y conseguir más apoyos. ${ }^{6}$ Por ejemplo, Enrique Peñalosa (exalcalde de la ciudad), quien había firmado el primer рот en el año 2000, el 31 de marzo de 2013, aprovechando una entrevista que estaba dando sobre política nacional y posibles coaliciones electorales para la contienda presidencial de 2014, se expresó también sobre la modificación que iba a discutirse dos meses después en el Concejo:

El alcalde ha contribuido a que incrementen terriblemente los precios de la vivienda popular en Bogotá, al congelar la tierra para urbanizar en la ciudad sin ningún conocimiento. Por ejemplo, al comienzo salió a decir que no se necesitaba mucha vivienda porque la población en Bogotá no estaba creciendo, cuando la demanda

6 Si bien este análisis no profundiza en la facilidad (o no) para que se integren nuevos actores a la coalición, es decir, si es una coalición promotora abierta o cerrada, investigaciones más profundas sobre este tema han sido realizadas por Vicente (2012). 
de vivienda tiene que ver con el crecimiento de hogares y no con la sobrepoblación. Entonces, Bogotá en los próximos 40 años va a crecer fácilmente 4 o 6 millones de habitantes y el tamaño de hogares será más pequeño. Entonces: con la misma población se necesitan más viviendas. (El Nuevo Siglo, 2013a)

La coalición promotora pro modificación haría lo mismo en prensa:

En Bogotá, los ciudadanos tenemos que movernos grandes distancias, unos pocos en carro, otros muchos en transporte público o a pie. Consolidamos un modelo irracional que no permite tener calidad de vida. Bordes llenos de edificaciones, cuando la mayor parte de la gente trabaja en el centro. Esto produce depredación y segregación social, desigualdad y violencia. El рот quiere cambiar eso. Según expertos, Bogotá tiene una de las "densidades" más grande del mundo, pero en Patio Bonito o en el Rincón de Suba. Matemáticamente, para que eso sea posible, es porque en casas de dos pisos -sin calles, parques o espacio público- viven cuatro o cinco familias. Lo que tenemos, entonces, está lejos de ser densificación; es hacinamiento. En cambio, nuestro centro es uno de los más desocupados del mundo: incluye 600 hectáreas de edificaciones vacías en Santa Fe, en Plaza España, en los Sanandresitos, en la zona industrial; 600 hectáreas cruzadas por las principales troncales de Transmilenio, la Caracas por el oriente, la 26 por el norte, la 30 por el sur, la Sexta, la Jiménez, todas cruzando un área deshabitada por las noches. Tenemos que cambiar esa lógica. (Petro, 2013)

Sin embargo, asegurar legitimidad al proyecto por parte de la ciudadanía no sería suficiente sin conocer que el mismo no estaba respaldado por políticos nacionales y gremios económicos con peso en el escenario nacional. La coalición statu quo estaba apoyada por el gobierno nacional, en especial por el vicepresidente Germán Vargas Lleras y el ministro de Vivienda, Luis Felipe Henao. Ambos, en reiteradas oportunidades, manifestaron su desacuerdo con la aprobación de la мерот у buscaron que su partido, Cambio Radical, hiciera oposición al proyecto. De igual forma, gerentes de los gremios nacionales estuvieron atentos a la discusión del proyecto en el Concejo:

(...) no solo se perdería suelo para reducir el déficit de vivienda, sino la posibilidad de desarrollar cerca de más de 54 millones de metros cuadrados y sigue disminuyendo el aporte de empleo de la construcción de la ciudad. [Entrevista a Martha Moreno sobre el impacto de la мерот, presidenta de Camacol, 3 de junio de 2013] (El Nuevo Siglo, 2013c)

Esto va a generar algo muy grave y es que se sigan perdiendo empleos, más de 79 mil puestos de trabajo se han perdido en la ciudad, se presentará un desplazamiento de la capacidad productiva de la capital a otras ciudades perdiendo ańos en el desarrollo de la misma. [Luis Felipe Henao, ministro de Vivienda, 29 de mayo de 2013] (El Nuevo Siglo, 2013b)

Gustavo Petro respondería al gobierno nacional anunciando su proyecto político nacional: "Cuando entré a la guerrilla, quise cambiar al país por fuera del Estado, y desde que dejamos las armas quiero cambiar al país desde el Estado. Para hacerlo, mi propósito inmediato es cambiar la ciudad" (El Tiempo, 2013, junio 15). Por lo 
anterior, la discusión que pudo ser técnica fue netamente política. El alcalde de la ciudad buscaba ser Presidente de la República posteriormente. Algunos miembros de la oposición también tenían su candidato, Germán Vargas Lleras, vicepresidente del gobierno de Juan Manuel Santos. Mostrar que el alcalde no producía resultados, que era ineficiente e improvisaba en el diseño de políticas públicas, fue una de las estrategias para ganar legitimidad respecto de la ilegalidad del proyecto.

Trasladar a la prensa el debate en torno al Plan de Desarrollo y Ordenamiento Territorial jugó a favor de la coalición promotora statu quo, pues creó la sensación de que la мерот no era un proyecto viable, carecía de motivaciones técnicas y no buscaba consolidar objetivos del Plan de Desarrollo vigente; lo mostró como un proyecto ideológico, que respondía a "caprichos" del alcalde por implementar medidas que, en la práctica, afectaban al sector constructor. Esta imagen se creó por distintas razones: i) por las diferencias entre el gobierno nacional y el local respecto de la localización de la vivienda social, y que mostraban a la alcaldía como inoperante para la producción de vivienda; ii) porque los gremios se movilizaron también por fuera del Concejo y la prensa hizo eco de sus inquietudes sobre el proyecto; y iii) también porque el alcalde tenía diferencias ideológicas con algunas líneas editoriales, lo que hizo difícil que el mensaje se trasmitiera con mayor fuerza por los medios:

Tuvimos un espacio de discusión política entre los ponentes. Sin embargo, en el Concejo no es un espacio donde lo técnico prima. Tú te basas en unas condiciones técnicas para tener una posición política, pero lo técnico te da para cualquier posición política. Entonces, técnicamente se dieron unas discusiones, cuando al final era política. (Diego García Bejarano, concejal Partido Progresista; com. pers., 17 de julio de 2018)

En esa línea, para modificar la discusión sobre la legitimidad del proyecto, la coalición statu quo entró a cuestionar las motivaciones y la sustentación técnica presentada por la alcaldía:

Yo no conozco nadie (...) que sea capaz de decir que hay que afectar el medioambiente en la construcción de la ciudad (...) ¿Que no hay cambio climático y elementos de gestión de riesgo? Todos lo sabemos [que existe]. Lo que pasa es que el cambio climático no se resuelve con normas urbanísticas en el territorio de Bogotá. Eso es mentira. Porque el análisis del cambio climático es regional, no por ciudad. Lo grave no es eso (...). Resulta, señores, que estamos cambiando toda la estructura ecológica principal de la ciudad (...) en todo el articulado se le otorgan facultades a todo el mundo para que, a través de actos administrativos, se modifique la eep [Estructura Ecológica Principal]. (Juan Manuel González, abogado urbanista. Sesión del Concejo del 24 de mayo, 2013)

Si bien existían los datos censales de 2005 y con base en ellos se proponía un ajuste poblacional a la dinámica urbana de la ciudad y, por tanto, la reducción de su zona de expansión, la administración distrital no presentó otro estudio técnico para justificar las modificaciones. Esto quedó aún más visible cuando se discutieron las modificaciones ambientales, pues si bien la Memoria Justificativa del proyecto incluía 
datos sobre variabilidad climática, temporadas de lluvias y el sistema de amenazas de la ciudad, carecía de soporte técnico-académico respecto de la relación entre estos datos y el cambio climático:

\begin{abstract}
Acerca de los artículos sobre la estructura ecológica, la gestión del riesgo y el cambio climático, no hay un solo estudio que sustente las conclusiones. Solo concluyen que tocará hacer dichos estudios. Debió haber sido al revés: que unos estudios ya hechos justifican modificar el рот. Nos van a meter en un limbo jurídico. Con el pretexto de que el cambio climático existe, lo cual es cierto, se van a poner a manipular el ordenamiento territorial. Claro que hay que prepararnos. Pero lo que está en este articulado indica que no tenemos los estudios ni los instrumentos y que, muy por el contrario, van a cambiar el рот actual, que es muy juicioso y tiene certezas jurídicas, algo que se les reconoce a tanto a Peñalosa como a Carmenza Saldías. [Concejal Miguel Uribe, 4 de junio, 2013] (Rueda, 2013)
\end{abstract}

Para la administración, sin embargo, ajustar el ordenamiento territorial a los desequilibrios ambientales recientes en la ciudad implicaba limitar el crecimiento acelerado hacia la periferia de la ciudad y aumentar las áreas de protección ambiental.

Es interesante que algunos aspectos que pudieron ser negociables (secundary aspects) o en los que inclusive concordaban ambas coaliciones (la legalización de los usos múltiples o la simplificación de normas urbanas) no fueron tenidos en cuenta en el debate y sí se abordó exclusivamente la (i)legalidad del proyecto, demostrando así que antes que acceder a cualquier tipo de negociación entre ambas coaliciones, era preferible rechazar el proyecto. En términos políticos, era importante-como así sucedió- crear un ambiente de desgobierno alrededor de la administración de Gustavo Petro.

Otros dos factores concurrieron en la transformación política del proyecto o shocks externos: por un lado, el proceso en curso de revocatoria al mandatario local, que buscaba apartarlo del cargo público y convocar a nuevas elecciones locales; y la discusión en el Concejo respecto de otros dos proyectos relevantes para la administración: la aprobación a un cupo de endeudamiento y la eliminación del cobro de valorización a varios proyectos urbanísticos de la ciudad. El primero, más político que el segundo, influyó en los concejales de oposición, sobre todo para hacer campańa política en contra del alcalde, reforzando aún más la idea de que la administración local no estaba cumpliendo sus promesas de campaña ni estaba ejecutando políticas públicas en beneficio de la ciudad, y sí en su proyecto político personal.

De igual forma, shocks internos influyeron en el trascurso del trámite normativo: la ausencia de operadores políticos que modularan la discusión, pues si bien el secretario de Gobierno asistió a algunos de los debates de la comisión y asistió a la votación del proyecto, la coordinación estuvo a cargo del secretario de Planeación, Gerardo Ardila, y de su equipo, en su mayoría personas del sector académico que por primera vez se enfrentaban a la gestión de trámites normativos. Esta inexperiencia también era compartida por los concejales progresistas del movimiento político del alcalde, que llegaban por primera vez al Concejo y que no conocían las reglas, tanto formales como informales, que imperan en el desarrollo de las discusiones. 
Finalmente, pese al rechazo del proyecto en el Concejo de la ciudad, la administración de Bogotá decidió expedirlo por decreto en septiembre de ese mismo año, 2013, trasladando el conflicto a otras esferas de poder, principalmente a lo que se ha denominado la "judicialización de la política", pues algunos de los actores de la coalición statu quo demandaron la aplicación de la norma ante el Consejo de Estado, pidiendo la suspensión y anulación del proyecto. En abril de 2014 quedarían suspendidos los efectos legales de la мерот, у el 20 de mayo de 2019, seis años después de haberse iniciado su discusión en el Concejo, se anularía el Decreto 364 de 2013 por medio del cual se modificaban excepcionalmente algunas normas urbanísticas de la capital.

\section{Consideraciones finales}

Este artículo identificó y describió las posiciones y creencias de los actores durante la discusión del proyecto de acuerdo de Modificación excepcional al Plan de Ordenamiento Territorial de Bogotá de 2013. Mostramos que se formaron dos coaliciones alrededor del proyecto y cómo ambas, con diferentes recursos y estrategias, buscaron posicionar sus creencias en el proyecto para que se modificara o no la política pública.

Como se ha analizado, la coalición statu quo tenía mayores recursos, entre los cuales se puede contar una estrategia nacional de desprestigio a la administración de Gustavo Petro, que lo mostraba como un gerente ineficiente e improvisador. A la vez, se señaló cómo el proyecto no se discutió técnicamente y sí se transformó en un asunto de medición de fuerzas políticas entre Gustavo Petro y Germán Vargas Lleras alrededor de la (i)legalidad del proyecto y de la discusión sobre un proyecto político nacional.

El proyecto que proponía hacer ajustes al Plan de Ordenamiento Territorial no contaba tampoco con el apoyo del gremio constructor de la ciudad -un actor relevante, dada la cantidad de recursos de que dispone-, por lo que se recurrió a desvirtuar sus aspectos técnicos. Como señalan Innes y Booher (2014), la argumentación técnica nunca es suficiente, pero sí es necesaria en la discusión de proyectos normativos. Este estudio de caso así lo ha corroborado.

Finalmente, es importante mencionar que, a pesar de haber voluntad política del ejecutivo local respecto de la modificación de políticas públicas, cualesquiera que sean, ello no siempre es suficiente en sistemas democráticos multinivel y donde las decisiones no son tomadas por un único actor público. Las coaliciones políticas y la negociación con los grupos de interés son más que necesarias en la aprobación de cualquier proyecto normativo. 


\section{Referencias bibliográficas}

Asociación Nacional de Instituciones Financieras (ANIF), Centro de Estudios Económicos. (2013, diciembre 12). La redensificación de Bogotá y la мерот. ANIF, Comentario económico del día. https://www.anif.com.co/sites/default/files/uploads/Dic12-13.pdf

Cairney, P. (2016). Paul A. Sabatier, "An advocacy coalition framework of policy change and the role of policy-oriented learning therein”. En M. Lodge, E. C. Page \& S. J. Balla (Eds.), The Oxford Handbook of Classics in Public Policy and Administration. Oxford, uK: Oxford University Press. https://doi.org/10.1093/oxfordhb/9780199646135.013.24

Concejo de Bogotá. (2013, mayo 19). Sesión 19 de mayo de 2013: “Arranca hoy en la Comisión del Plan de Concejo de Bogotá la presentación de la Administración Distrital del proyecto de acuerdo para modificar el РОт", por H. C. Javier Palacio. Bogotá. https:// bit.ly/3gW1asM

Congreso de la República, Colombia. (1997). Ley 388 de 1997 (julio 18). Por la cual se modifica la Ley 9a de 1989, y la Ley $3^{\text {a }}$ de 1991 y se dictan otras disposiciones. Diario Oficial núm. 43.091, de 24 de julio de 1997. https://www.minambiente.gov.co/ images/normativa/leyes/1997/ley_0388_1997.pdf

Cortez, M. \& Maillet, A. (2018). Trayectoria multinivel de una coalición promotora e incidencia en la agenda política nacional. El caso del conflicto de Pascua Lama y la ley de glaciares en Chile. Colombia Internacional, (94), 3-25. http://dx.doi.org/10.7440/ colombiaint94.2018.01

Consejo Territorial de Planeación Distrital, Bogotá. (2013). Concepto del Consejo Territorial de Planeación Distrital sobre el proyecto de acuerdo: "Modificación Excepcional de normas urbanísticas del рот 2013”. Bogotá: Consejo Territorial de Planeación Distrital (СтPD). https://bit.ly/3bW61Zf

El Nuevo Siglo. (2013a, marzo 31). Verdes no deberían estar en Unidad Nacional: Peñalosa. El Nuevo Siglo, pp. 9A-10A-11A. https://issuu.com/elnuevosiglo/docs/domingo_31_ marzo_7_abril

El Nuevo Siglo. (2013b, mayo 29). Bogotá: Construcción de vivienda vs. Рот. El Nuevo Siglo, p. 15A. https://www.elnuevosiglo.com.co/index.php/articulos/5-2013-bogotaconstruccion-de-vivienda-vs.-pot

El Nuevo Siglo. (2013c, junio 3). Quiero reforma del рот por acuerdo del Concejo: Alcalde. El Nuevo Siglo, p. 15A. https://issuu.com/sebashmora/docs/el_nuevo_siglo

El Tiempo. (2013, junio 15). 'Si no hay fraude, no habrá revocatoria de mi mandato': Gustavo Petro. El Tiempo, p. B61. https://www.eltiempo.com/archivo/documento/CMS12873062

Hernández Mora, S. (2019). La reparación integral a las víctimas del conflicto armado en Colombia: un análisis de la construcción de la Ley de Victimas y Restitución de Tierras (2011) a través del Advocacy Coalition Framework (ACF). Tesis de maestría, Gobierno y Asuntos Públicos, Universidad Nacional Autónoma de México. https://bit.ly/33s5B95

Innes, J. \& Booher, D. (2014). A turning point for planning theory? Overcoming dividing discourses. Planning Theory, 14(2), 195-213. https://doi. org/10.1177\%2F1473095213519356 
Jaramillo, S. \& Cuervo, N. (2013). Colombia: un caso paradigmático de la política neoliberal de vivienda en América Latina. Presentación en Seminario Internacional: A cidade neoliberal na América Latina. Rio de Janeiro, 6-8 de noviembre, 2013.

Jaramillo, S. \& Cuervo, N. (2014). Precios inmobiliarios de vivienda en Bogotá 1970-2013. Bogotá: Ediciones Uniandes, Facultad de Economía, Centro de Estudios sobre Desarrollo Económico (CEDE).

Jenkins-Smith, H., Silva, C. L., Gupta, K. \& Ripberger, J. T. (2014). Belief system continuity and change in policy advocacy coalitions: Using cultural theory to specify belief systems, coalitions, and sources of change. Policy Studies Journal, 42(4), 484-508. https://doi.org/10.1111/psj.12071

Labiano, V. I. (2018). Cannabis medicinal en Argentina: Cambio menor en el subsistema de política de drogas. Revista Uruguaya de Ciencia Politica, 27(2), 75-98. http://dx.doi. org/10.26851/rucp. 28.2

Leal, J. (2002). Segregación social y mercados de vivienda en las grandes ciudades. Revista Española de Sociología, (2), 59-75. https://recyt.fecyt.es/index.php/res/article/ view/64866

Marques, E. \& Kowarick, L. (2013). São Paulo: novos percursos e atores: sociedade, cultura e politica. São Paulo: Editora 34

Meglio, M. S. \& Pluchino, J. L. (2019). Ideas, actores e intereses en disputa: Análisis del Programa de Desarrollo Rural Sustentable en el Partido de General Pueyrredón. Revista Pilquen, 22(2), 39-49. https://dialnet.unirioja.es/servlet/articulo?codigo=7008162

Pérez Zorrilla, J. (2016). Acuerdos y tensiones dentro de la izquierda política uruguaya en materia de educación media: Un aporte desde el marco de las coaliciones promotoras. Revista Uruguaya de Ciencia Política, 25(2), 35-55. https://www.redalyc.org/articulo. oa?id=297349042002

Petro, G. (2013, marzo 17). El Plan de Ordenamiento Territorial. Dos maneras de entender el mundo. El Tiempo, pp. D34, B61. https://issuu.com/sebashmora/docs/el_tiempo

Pierce, J. J., Peterson, H. L. \& Hicks, K. C. (2017). Policy change: An advocacy coalition framework perspective. Policy Studies Journal, 48(1), 64-86. https://doi.org/10.1111/ psj. 12223

Presidencia de la República, Colombia. (2004, noviembre 30). Decreto Nacional 4002 de 2004. Por el cual se reglamentan los artículos 15 y 28 de la Ley 388 de 1997. Bogotá: Ministerio de Medio Ambiente, Vivienda y Desarrollo Territorial. https://diariooficial.vlex.com.co/vid/decreto-352555178

Rueda, M. I. (2013, junio 4). ¿El рот de Petro es un planteamiento ideológico? El Tiempo, pp. D34, B63. https://issuu.com/sebashmora/docs/el_tiempo_2

Sabatier, P. \& Jenkins-Smith, H. (1999). The advocacy coalition framework: An assessment. En P. Sabatier (Ed.), Theories of the Policy Process (pp. 117-166). Boulder, co: Westview Press.

Sabatier, P. \& Weible, C. (2007). The advocacy coalition framework: Innovations and clarifications. En P. Sabatier (Ed.), Theories of the Policy Process (pp. 189-220). Boulder, co: Westview Press.

Sabatier, P. \& Weible, C. (2010). El marco de las coaliciones promotoras. En Teorías del proceso de las políticas públicas (pp. 203-238). Ciudad Autónoma de Buenos Aires: Presidencia de la Nación. 
Sabatini, F. \& Brain, I. (2008). La segregación, los guetos y la integración social urbana: mitos y claves. EURE, 34(103), 5-26. http://dx.doi.org/10.4067/S0250-71612008000300001

Sánchez Ospina, A. (2019). El comportamiento político en la toma de decisiones públicas: el caso de la expedición de la Modificación excepcional del Plan de Ordenamiento Territorial de Bogotá, 2013. Tesis de maestría, Estudios Políticos y Sociales, Universidad Nacional Autónoma de México, México. https://bit.ly/32vqWz8

Sanjurjo García, D. (2013). La aplicación del enfoque de coaliciones promotoras al cambio en las políticas de drogas en el Uruguay. Documento para su presentación en el IV Congreso Internacional en Gobierno, Administración y Politicas Públicas GIGAPP-IUIOG. Instituto Nacional de Administración Pública (Madrid, España), 23 y 24 de septiembre 2013. https://bit.ly/2Cr2ZPd

Secretaría Distrital de Planeación, Bogotá. (2011). Encuesta multipróposito del 2011. Bogotá: Secretaría Distrital de Planeación. https://bit.ly/2DSFrUd

Secretaría Distrital de Planeación, Bogotá. (2013). Modificación Excepcional de Normas Urbanisticas del Plan de Ordenamiento Territorial 2013. Memoria Justificativa / Documento resumen. Bogotá: Secretaría Distrital de Planeación. https://issuu.com/ sebashmora/docs/memoria_justificativa_mepot 